\title{
Interaction of rule and attribute learning in in identification of concepts involving binary rules
}

\author{
IRWIN D. NAHINSKY, ARWA AAMIRY, and RICHARD M. BAIRD \\ University of Louisville, Louisville, Kentucky 40208
}

\begin{abstract}
An experiment was performed in which subjects were required in certain problems to discover which of two binary rules, conjunctive or exclusive disjunctive, was correct. In addition, they were required to discover which two attributes were relevant. Subjects were given direct information about the correct rule either on the first or sixth trial and received varying degrees of information about the potentially correct hypothesis on the first six trials. In some problems, possible solutions were narrowed to two, one for each binary rule, by Trial 6 . In other problems, less information was given during these trials. It was found that subjects used rule information to solve the problems, and that hypothesis information was helpful in the absence of information about the correct binary rule. Evidence indicated that subjects tracked hypotheses involving both types of rules simultaneously.
\end{abstract}

Much concept learning research has been concerned with the processes whereby subjects identify the correct attributes specified by a known binary rule, such as conjunction or disjunction. Bourne (1970), and Haygood and Bourne (1965) have focused attention upon the need to study the strategies whereby the correct binary rule is discovered. Various theories have been formulated to conceptualize strategies which might be used by a subject to organize his search for correct rules. Neisser and Weene (1962) categorized rules with two relevant values in a heirarchical scheme in which the number of binary operations making up the rule determined its level of complexity. They hypothesized that learning proceeds from lower levels of complexity to higher levels, whereby subjects start with simpler rules and proceed to more complex rules of which the simpler rules are components. Hunt (1962) developed an elaborate decision tree approach to conceptualize the process.

Although research has emphasized either rule or attribute learning, little attention has been paid to the problems encountered when subjects must discover both the correct attributes and the rules combining them. Several questions are appropriate in this context. Do subjects search simultaneously for the correct attributes and the rule combining them or must they have some sort of working hypothesis about a potentially correct rule to organize their search for correct attribute values? In view of the fact that different strategies are appropriate for different

The research was supported in part by NIMH grant MH20322-01. The authors would like to acknowledge Gertrude Nahinsky for her assistance in the data analysis. Requests for reprints should be sent to Irwin D. Nahinsky, Psychology Department, University of Louisville, Louisville, Kentucky 40208. binary rules, e.g., conjunctions and inclusive disjunctions, the latter possibility would seem more likely. Can subjects use attribute value information before they have settled upon a rule to organize their search? Do they store potentially useful information to be used when incorrect rules are finally eliminated?

The experiment reported here was designed to explore questions raised above. The subjects were presented with problems in which the rule to be discovered involved a combination of two attribute values, also to be discovered. The correct rule was either a conjunction or an exclusive disjunction. Information about the correct rule was given directly for some problems either early or late in the stimulus series. This was done to insure that the subject did indeed receive correct rule information at specific points in learning. This procedure was also helpful in assessing the extent to which a subject could use rule information when it was presented in the stimulus series The two rules, conjunction and exclusive disjunction, proved to be suitable in that they required different strategies. These rules also allowed the experimenters to present information eliminating many hypotheses for both rules in a reasonably small number of trials.

\section{METHOD}

\section{Subjects}

The subjects were 32 students in introductory psychology whose participation fulfilled a course requirement.

\section{Stimuli}

Stimuli were geometric figures printed on glossy 3 by 5 in. cards. The patterns on the stimuli varied with respect to three dimensions: (a) shape of figures - crosses, circles, or squares; (b) shading of figures-black, striped, or white; (c) number of figures-1, 2, or 3.

\section{Design}

Each subject was presented with two successive problems. The first of the two problems required a conjunctive solution ("striped 
circles") and the second an exclusive disjunctive solution ("white or cross but not both"). These two problems were presented under two rule-type information conditions. One-half of the 32 subjects were told at the beginning of each of the two problems what the correct rule type was and the other half were given this information following Trial 6 . Within each of these groups of 16 subjects, two presentation conditions were balanced with the two problems in a Latin square design. For one condition, the first six stimuli contained information jointly which eliminated all but two possible solutions, one conjunctive and the other exclusive disjunctive. This condition will be termed the greater-hypothesis-information (GHI) condition. For the other condition, the lesser-hypothesisinformation (LHI) condition, the first six stimuli were merely a random subset of the 27 stimuli in the population. Thus, there were four groups of eight subjects each, with two Latin square designs, one for direct rule information early (RIE) and the other for direct rule information late (RIL). All subjects received the remaining 21 stimuli in random order, and cycles of 27 stimuli were repeated until the subject reached criterion, unless criterion was not reached at the end of four cycles, whereupon the subject was terminated on the problem. Subjects had to classify stimuli as exemplars or nonexemplars of the concept. The subject was run to a criterion of 10 consecutive correct responses and then asked to state the solution. An incorrect statement was followed by another run to criterion.

\section{Procedure}

Subjects were assigned upon appearance to presentation order groups in a random fashion. Each subject was seated at the table in a distraction-free, enclosed room. The experimenter read the instructions with the subject. The task was explained with the aid of a diagram showing all possible stimulus patterns. The subject was told that the solution to each problem depended upon two of the three dimensions and that the rule combining the two relevant values could be one of two types-conjunctive (explained as an "and" rule) or exclusive disjunctive (explained as an "or" rule), with each rule illustrated by examples from the stimulus set. Each subject was instructed that he might have to discover the correct rule and would be required to learn the two values associated with the rule in each problem. (Subjects received some additional problems after the experiment which required rule discovery for subjects in all conditions.) Thus, the instructions were similar to those of the usual attribute identification studies for each subtype, with additional instructions for rule identification.

A screen separated experimenter and subject during the session. A subject was handed each stimulus card in turn whereupon he made his classification as indicated by the instructions. The experimenter gave feedback about the correctness of the choice after each stimulus presentation. Each subject was given two practice problems, one involving each binary rule as a correct solution. The subjects were run to a criterion of 10 correct responses or until they could state the type of rule that was appropiate. Stimulus sequences on these problems were fixed so that information determining the problem type was given early in the series. Half of the subjects received one problem first and half received the other first in a randomly determined manner.

After completion of the practice problems and clarification of procedures, each subject was then given each experimental problem in turn as specified by his condition. A diagram containing all possible stimuli was available to subjects throughout the experiment.

\section{RESULTS AND DISCUSSION}

Analyses of variance were performed for both total errors and for trials to criterion, with means for significant sources of variation shown for both conditions in Table 1 . The analysis of variance of results for errors paralleled almost exactly that for
Table 1

Mean Errors and Mean Trials to Criterion for Conditions Representing Significant Sources of Variation $(N=32)$

\begin{tabular}{|c|c|c|c|c|}
\hline & \multicolumn{2}{|c|}{\begin{tabular}{l}
\multicolumn{1}{c}{ Rule } \\
Information \\
Early (RIE)
\end{tabular}} & \multicolumn{2}{|c|}{$\begin{array}{c}\text { Rule } \\
\text { Information } \\
\text { Late (RIL) }\end{array}$} \\
\hline & GHI* & LHI** & GHI* & LHI** \\
\hline Mean Errors & 10.25 & 9.31 & 6.31 & 16.81 \\
\hline Mean Trials to Criterion & 34.38 & 29.81 & 26.69 & 41.88 \\
\hline $\begin{array}{l}\text { Mean Errors } \\
\text { Mean Trials to Criterion }\end{array}$ & $\begin{array}{r}\text { Conju } \\
2\end{array}$ & $\begin{array}{l}\text { nctive } \\
.44 \\
.34\end{array}$ & $\begin{array}{r}\text { Disju } \\
14 \\
42\end{array}$ & $\begin{array}{l}\text { ctive } \\
91 \\
03\end{array}$ \\
\hline
\end{tabular}

*Reduction to two hypotheses on first six trials.

**Random hypothesis information on first six trials.

trials to criterion. Neither rule information nor hypothesis information produced a significant main effect. However, there was a signficant interaction between rule information and hypothesis information, $\mathrm{F}(1,28)=5.03, \mathrm{p}<.05$. It is evident that the RIL-LHI condition produced markedly more errors than did the other three conditions. A contrast of this mean with the other three means produced a highly significant effect, $\mathrm{F}(1,28)=7.72, \mathrm{MS}_{\mathrm{e}}=78.11$, $p<.01$. In addition, the means were contrasted using the Newman-Keuls procedure with comparison between the two RIL conditions producing the only significant effect, $\mathrm{q}=4.12, \mathrm{SE}=2.55, \mathrm{p}<.05$. Thus, subjects showed improved performance when the stimulus series contained a great deal of information limiting potentially correct hypotheses of either rule type, even if this information was presented before they had the information specifying the correct rule type. In contrast, a comparison of the two hypothesis-limiting conditions for the RIE condition did not produce a significant result. Results then showed that subjects receiving information limiting potentially correct hypotheses to two during the first six trials do about as well when they receive information specifying the correct hypothesis after Trial 6 as when this information is given before the first trial. The complete lack of interaction between rule type and any of the other variables indicated that such effects are not specific to one rule type or the other. There was a significant main effect for rule type with disjunctive problems producing significantly more errors, $F(1,28)=11.02, p<.01$.

The interaction between trial of direct rule information and amount of specific hypothesis information on the first six trials brings to mind some possible interpretations. One might hypothesize that when the subject is not informed about the correct binary rule, he adopts some working hypothesis about a rule and eliminates attribute-value pairings not consistent with stimulus information under the assumption of that rule. With efficient use of maximum hypothesis information preceding rule 
information, subjects able to choose the correct rule initially would solve the problem immediately upon being informed of the correct rule. However, only those fortunate subjects would benefit from the strategy. The fact that subjects in the favorable hypothesis-information condition did as well in the late rule-information condition as in the early rule-information condition makes the preceding interpretation difficult to defend. It appears that efficiency of use of information about potentially correct hypotheses of both rule types is independent of direct availability of the correct rule type. Subjects in the RIL condition apparently tracked hypotheses associated with both rules simultaneously. Such a strategy might be expected in view of the fact that information favoring one rule or the other is not available before Trial 6 in this condition. In view of the fact that the hypotheses remaining by Trial 6 involved different value pairs for the two rules, a simple strategy in which the subject organizes a rule search around tentative value pairs may not provide an adequate explanation of the results. The fact that the two rules are of different levels of complexity according to Neisser and Weene (1962) makes the result interesting. Conjunction is a binary rule of Level II, while exclusive disjunction, a disjunction of two conjunctions, is of Level III. The results suggest that two rules of different complexity levels are tracked simultaneously rather than successively as suggested by Neisser and Weene.

\section{REFERENCES}

Bourne, L. E., JR. Knowing and using concepts. Psychological Review, 1970, 77, 546-556.

Bruner, J. S., Goodnow, J. J., \& Austin, G. A. A study of thinking. New York: Wiley, 1956.

HAYGOOD, R. C., \& Bourne, L. E., JR. Attributeand rule-learning aspects of conceptual behavior. Psychological Review, 1965, 72, 175-195.

Hunt, E. B. Concept learning: An information processing problem. New York: Wiley, 1962.

Hunt, E. B., Marin, J. M., \& Stone, P. Experiments in induction. New York: Academic Press, 1966.

Neisser, U., \& WEENE, P. Heirarchies in concept attainment. Journal of Experimental Psychology, 1962, 64, 644-645.

(Received for publication October 9, 1975.) 